\title{
DYER-LASHOF OPERATIONS IN $K$-THEORY
}

\author{
JAMES E. MCCLURE 1
}

Dyer-Lashof operations were first introduced by Araki and Kudo in [1] in order to calculate $H_{*}\left(\Omega^{n} S^{n+k} ; Z_{2}\right)$. These operations were later used by Dyer and Lashof to determine $H_{*}\left(Q Y ; Z_{p}\right)$ as a functor of $H_{*}\left(Y ; Z_{p}\right)$ [5], where $Q Y=\bigcup_{n} \Omega^{n} \Sigma^{n} Y$. This has had many important applications. Hodgkin and Snaith independently defined a single secondary operation in $K$-homology (for $p$ odd and $p=2$ respectively) which was analogous to the sequence of DyerLashof operations in ordinary homology $[7,13]$, and this operation has been used to calculate $K_{*}\left(Q Y ; Z_{p}\right)$ when $Y$ is a sphere or when $p=2$ and $Y$ is a real projective space $[\mathbf{1 1}, \mathbf{1 2}]$. In this note we describe new primary Dyer-Lashof operations in $K$-theory which completely determine $K_{*}\left(Q Y ; Z_{p}\right)$ in general.

We shall remove the indeterminacy of the operation by lifting it to higher torsion groups. First we establish notation. $X$ will always denote an $E_{\infty}$ space [9] and $Y$ will denote an arbitrary space, considered as a subspace of $Q Y$ via the natural inclusion. We write $K_{*}(Y ; r)$ for $K_{0}\left(Y ; Z_{p^{r}}\right) \oplus K_{1}\left(Y ; Z_{p^{r}}\right)$; in particular $K$-theory is $Z_{2}$-graded and we write $|x|$ for the mod 2 degree of $x$. There are evident natural maps

$$
\begin{gathered}
p_{*}^{s}: K_{\alpha}(Y ; r) \rightarrow K_{\alpha}(Y ; r+s) \text { if } s \geq 1, \\
\pi: K_{\alpha}(Y ; r) \rightarrow K_{\alpha}(Y ; t) \text { if } 1 \leq t \leq r,
\end{gathered}
$$

and

$$
\beta_{r}: K_{\alpha}(Y ; r) \rightarrow K_{\alpha-1}(Y ; r) .
$$

THEOREM 1. For each $r \geq 2$ and $\alpha \in Z_{2}$ there is an operation

$$
Q: K_{\alpha}(X ; r) \rightarrow K_{\alpha}(X ; r-1)
$$

with the following properties, where $x, y \in K_{*}(X ; r)$.

(i) $Q$ is natural with respect to $E_{\infty}$-maps.

$$
Q(x+y)=\left\{\begin{array}{l}
Q x+Q y-\pi\left[\sum_{i=1}^{p-1} \frac{1}{p}\left(\begin{array}{l}
p \\
i
\end{array}\right) x^{i} y^{p-i}\right] \\
Q x+Q y \quad \text { if }|x|=|y|=1 .
\end{array} \text { if }|x|=|y|=0,\right.
$$

(iii) $Q \phi=0$, where $\phi \in K_{0}(X ; r)$ is the identity element.

$$
Q(x y)=\left\{\begin{array}{l}
Q x \cdot \pi\left(y^{p}\right)+\pi\left(x^{p}\right) \cdot Q y+p(Q x)(Q y) \text { if }|x|=|y|=0 \\
Q x \cdot \pi\left(y^{p}\right)+p(Q x)(Q y) \text { if }|x|=1,|y|=0 \\
(Q x)(Q y) \text { if }|x|=|y|=1
\end{array}\right.
$$

Received by the editors August 16, 1982 and, in revised form, September 21, 1982. 1980 Mathematics Subject Classification. Primary 55N15, 55S12.

${ }^{1}$ Research partially supported by NSF grant MCS-8018626 .

(C) 1983 American Mathematical Society 0273-0979/82/0000-1039/\$02.25 


$$
\sigma Q x=\left\{\begin{array}{l}
Q \sigma x \text { if }|x|=0, \\
\pi(\sigma x)^{p}+p Q \sigma x \text { if }|x|=1,
\end{array}\right.
$$

where $\sigma: \tilde{K}_{\alpha}(\Omega X ; r) \rightarrow K_{\alpha+1}(X ; r)$ is the homology suspension.

(vi) If $k$ is prime to $p$, then $Q \psi^{k}=\psi^{k} Q$, where $\psi^{k}$ is the $k$ th Adams operation.

$$
\beta_{r-1} Q x= \begin{cases}Q \beta_{r} x-p \pi\left(x^{p-1} \beta_{r} x\right) & \text { if }|x|=0 \\ \pi\left(\beta_{r} x\right)^{p}+p Q \beta_{r} x & \text { if }|x|=1\end{cases}
$$

$$
\begin{aligned}
& Q \pi x=\pi Q x \text { if } r \geq 3 \text { and } \\
& \qquad p_{*} x=\left\{\begin{array}{l}
x^{p} \text { if }|x|=0, r=1, \\
p_{*} Q x-\left(p^{p-1}-1\right) x^{p} \quad \text { if }|x|=0, r \geq 2, \\
0 \text { if }|x|=1, r=1, \\
p_{*} Q x \text { if }|x|=1, r \geq 2 .
\end{array}\right.
\end{aligned}
$$

(ix) Let $p=2$. If $x \in K_{1}(X ; 1)$ then $Q \beta_{2} 2_{*} x=x^{2}$. If $x \in K_{1}(X ; 2)$ then $(\pi x)^{2}=\left(\pi \beta_{2} x\right)^{2} ;$ in particular $(\pi x)^{2} \in K_{0}(X ; 1)$ is zero if $x \in K_{1}(X ; r)$ with $r \geq 3$.

REMARKS. (i) There are no Adem relations.

(ii) If $x \in K_{*}(X ; 1)$ has $\beta x=0$ then $x$ lifts to $y \in K_{*}(X ; 2)$. Thus one can define a secondary operation $\bar{Q}$ on $\operatorname{ker} \beta$ by $\bar{Q} x=Q y$. The element $y$ is well defined modulo the image of $p_{*}$, and thus Theorem 1 (viii) shows that $\bar{Q} x$ is well defined modulo $p$ th powers if $|x|=0$ and has no indeterminacy if $|x|=1$. This is essentially the operation defined by Hodgkin and Snaith (although their construction is incorrect when $p$ is odd, as shown in [10]).

The next result shows that, in contrast to ordinary homology, $K_{*}(Q Y ; 1)$ will in general have nilpotent elements.

THEOREM 2. $\pi\left(\beta_{r} x\right)^{p^{r}}=0$ in $K_{0}(X ; 1)$ if $x \in K_{1}(X ; r)$.

If $x \in K_{*}(Y ; r)$, we write $Q^{s} x \in K_{*}(Q Y ; r-s)$ for the $s$ th iterate of $Q$ when $s<r$. These elements give a family of indecomposable generators in $K_{*}(Q Y ; 1)$, but in general there can be other generators as well. For example, if $x \in K_{1}(Y ; 1)$ with $\beta x \neq 0$ then $x(\beta x)^{p-1}$ has zero Bockstein by Theorem 2 , hence it lifts to an element $z \in K_{1}(Q Y ; 2)$, and it turns out that $Q z$ is indecomposable (note that we cannot apply the Cartan formula to $Q z$ ). The next theorem allows us to deal systematically with elements like $z$; in particular it gives the higher Bocksteins of such elements.

THEOREM 3. For each $r \geq 1$ there is an operation

$$
R: K_{1}(X ; r) \rightarrow K_{1}(X ; r+1)
$$

with the following properties, where $x, y \in K_{1}(X ; r)$.

(i) $R$ is natural for $E_{\infty}$-maps.

(ii) $p_{*} R x=R p_{*} x, \pi R x=Q p_{*} x-x\left(\beta_{r} x\right)^{p-1}$, and if $r \geq 2, R \pi x=Q p_{*} x-$ $p^{p-1} x\left(\beta_{r} x\right)^{p-1}$.

(iii) $\beta_{r+1} R x=Q \beta_{r+2} p_{*}^{2} x$.

(iv) If $r \geq 2$, then $Q R x=R Q x$. 
(v) If $k$ is prime to $p$, then $R \psi^{k}=\psi^{k} R$.

$$
\begin{gathered}
\sigma R x=\left\{\begin{array}{l}
p_{*}\left[(\sigma x)^{p}\right] \quad \text { if } r=1, \\
p_{*}\left[(\sigma x)^{p}\right]+p_{*}^{2} Q \sigma x \quad \text { if } r \geq 2 .
\end{array}\right. \\
R(x+y)=R x+R y-\sum_{i=1}^{p-1}\left[\frac{1}{p}\left(\begin{array}{c}
p \\
i
\end{array}\right)\left(p_{*} x\right)\left(\beta_{r+1} p_{*} x\right)^{i-1}\left(\beta_{r+1} p_{*} y\right)^{p-i}\right. \\
\left.+\left(\begin{array}{c}
p-1 \\
i
\end{array}\right) \beta_{r+1} p_{*}(x y)\left(\beta_{r+1} p_{*} x\right)^{i-1}\left(\beta_{r+1} p_{*} y\right)^{p-i-1}\right] .
\end{gathered}
$$

Theorems 1 and 3 imply that $\pi Q^{s} R^{t} x$ is decomposable if $x \in K_{1}(Y ; r)$ and $s<r+t-1$. If $s=r+t-1$ and $\pi \beta_{r} x \neq 0$ then this element turns out to be indecomposable.

In order to give a Cartan formula for $R$ and to provide generators for the higher terms of the Bockstein spectral sequence, we next give a $K$-theory analogue for the Pontryagin $p$ th power introduced in ordinary homology by Madsen [8] and May [4]. Note, however, that by part (viii) of the following theorem this operation does not give rise to new families of indecomposables in $K_{*}(Q Y ; 1)$.

THEOREM 4. For each $r \geq 1$ there is an operation

$$
\text { Q: } K_{0}(X ; r) \rightarrow K_{0}(X ; r+1)
$$

with the following properties.

(i) $\mathcal{Q}$ is natural for $E_{\infty}$-maps.

(ii) $\pi \mathcal{Q} x=x^{p}$ and $\mathcal{Q} p_{*} x=p^{p-1} p_{*} \mathcal{Q} x$. If $r \geq 2$ then $\mathcal{Q} \pi x=x^{p}$.

(iii) $\pi \beta_{r+1} \mathcal{Q} x=x^{p-1} \beta_{r} x$.

(iv) Let $p$ be odd. Then

$$
\begin{aligned}
& R(x y)=\left\{\begin{array}{l}
(R x)(\mathcal{Q} y) \text { if }|x|=1,|y|=0 \text { and } r=1, \\
(R x)(\mathcal{Q} y)+p_{*}^{2}[(Q x)(Q y)] \text { if }|x|=1,|y|=0 \text { and } r \geq 2 .
\end{array}\right. \\
& \mathcal{Q}(x y)=(\mathcal{Q} x)(\mathcal{Q} y) \text { if }|x|=|y|=0 .
\end{aligned}
$$

(v) If $k$ is prime to $p, \psi^{k} \mathcal{Q}=\mathcal{Q} \psi^{k}$.

$$
\mathcal{Q}(x+y)=\mathcal{Q} x+\mathcal{Q} y+\sum_{i=1}^{p-1} \frac{1}{p}\left(\begin{array}{l}
p \\
i
\end{array}\right) p_{*}\left(x^{i} y^{p-i}\right) \text {. }
$$

$$
\sigma \mathcal{Q} x=\left\{\begin{array}{l}
0 \quad \text { if } p \text { is odd } \\
2^{r-1} 2_{*}\left[(\sigma x)\left(\beta_{r} \sigma x\right)\right] \text { if } p=2 .
\end{array}\right.
$$

$$
Q \mathcal{Q} x=\left\{\begin{array}{l}
0 \quad \text { if } r=1, \\
\sum_{i=1}^{p}\left(\begin{array}{l}
p \\
i
\end{array}\right) p^{i-2} x^{p^{2}-i p} p_{*}\left[(Q x)^{i}\right] \quad \text { if } r \geq 2
\end{array}\right.
$$


REMARK. The formulas in part (iv) have analogues when $p=2$, but some of the coefficients in this case have not yet been determined.

Using the operations $Q$ and $R$ we can completely describe $K_{*}(Q Y ; 1)$. We shall assume that $Y$ is a finite complex, although this condition can be avoided. First recall the construction $C Y$ from [9]. By [4, Theorem I.5.10] we have $K_{*}(Q Y ; 1) \cong\left(\pi_{0} Y\right)^{-1} K_{*}(C Y ; 1)$, and so it suffices to give $K_{*}(C Y ; 1)$.

Next recall the reduced $K$-theory Bockstein spectral sequence $E_{*}^{r} Y$ from [2]. If $Y$ is a finite complex we have $E_{*}^{n} Y=E_{*}^{\infty} Y$ for some $n$, and we can choose a subset $A_{\infty} \subset \tilde{K}_{*}(Y ; Z)$ projecting to a basis for $E_{*}^{\infty} Y$. Proceeding inductively, we can choose subsets $A_{r} \subset \tilde{K}_{*}(Y ; r)$ such that

$$
A_{\infty} \cup A_{n-1} \cup \beta_{n-1}\left(A_{n-1}\right) \cup \cdots \cup A_{r} \cup \beta_{r}\left(A_{r}\right)
$$

projects to a basis of $E_{*}^{r} Y$ for $1 \leq r \leq n-1$. We write $A_{r 0}$ and $A_{r 1}$ for the zero- and one-dimensional subsets of $A_{r}$. Let $B Y$ be the quotient of the free strictly commutative algebra generated by the four sets

$$
\begin{gathered}
\left\{\pi Q^{s} x \mid x \in A_{r}, 0 \leq s<r\right\}, \quad\left\{\pi \beta_{r-s} Q^{s} x \mid x \in A_{r 0}, 0 \leq s<r<\infty\right\}, \\
\left\{Q^{r+s} R^{s+1} x \mid x \in A_{r 1}, r<\infty, 0 \leq s\right\}, \quad \text { and }\left\{\pi \beta_{r+s} R^{s} x \mid x \in A_{r 1}, r<\infty, 0 \leq s\right\}
\end{gathered}
$$

by the ideal generated by the set

$$
\left\{\left(\pi \beta_{r+s} R^{s} x\right)^{p^{r+s}} \mid x \in A_{r 1}, r<\infty, 0 \leq s\right\} .
$$

The Dyer-Lashof operations $Q$ and $R$ give an additive homomorphism $\lambda: B Y \rightarrow$ $K_{*}(C Y ; 1)$, which is a ring homomorphism if $p$ is odd but not if $p=2$. Our main theorem is

THEOREM 5. $\lambda$ is an isomorphism.

REMARKS. (i) Theorems 1,3 , and 5 also give the ring structure of $K_{*}(C Y ; 1)$ when $p=2$. First recall that $\bmod 2 K$-theory is noncommutative [2], in fact the commutator of $x$ and $y$ is $(\beta x)(\beta y)$. Now

$$
\beta\left(Q^{r+s} R^{s+1} x\right)=\left(\beta_{r+s+1} R^{s+1} x\right)^{2^{r+s}}
$$

if $x \in A_{r 1}$ with $r<\infty$ and $s \geq-1$, and all other generators (except $Q^{r-1} x$ for $x \in A_{r 0}, r<\infty$, whose Bockstein is the generator $\beta Q^{r-1} x$ ) have zero Bockstein and hence lie in the center. Further, all odd-dimensional generators have square zero except in the following cases:

$$
\begin{gathered}
\left(\pi Q^{r-2} x\right)^{2}=\left(\beta_{r} x\right)^{2^{r-1}} \text { if } x \in A_{r 1}, 2 \leq r<\infty \\
\left(Q^{r+s} R^{s+1} x\right)^{2}=\left(\pi \beta_{r+s+2} R^{s+2} x\right)^{2^{r+s}} \text { if } x \in A_{r 1}, r<\infty, s \geq-1 .
\end{gathered}
$$

These facts, together with Theorem 5 , determine the ring structure.

(ii) The effect of $(Q f)_{*}: K_{*}(Q Y ; 1) \rightarrow K_{*}(Q Z ; 1)$ for any $f: Y \rightarrow Z$ can be ascertained from Theorems 1,3 , and 5 if $f_{*}: K_{*}(Y ; r) \rightarrow K_{*}(Z ; r)$ is known for all $r \geq 1$ (although the formulas can become complicated unless $f_{*}$ takes the chosen sets $A_{r}$ for $Y$ into the corresponding sets for $Z$ ). In particular if $f: S^{2} \rightarrow S^{2}$ is the degree $p$ map then Theorem 1 (ii) implies that $(Q f)_{*}$ is nonzero on $K_{*}\left(Q S^{2} ; 1\right)$. Thus $K_{*}(Q Y ; 1)$ is not a functor of $K_{*}(Y ; 1)$, a fact first noticed by Hodgkin [7]. 
(iii) Theorem 5 specializes to give an independent proof of the computations of Hodgkin [6] and Miller and Snaith [11, 12]. The operation $R$ did not arise in those computations since in the cases considered $A_{r 1}$ was empty for all $r<\infty$.

Finally, we describe the Bockstein spectral sequence for $C Y$.

THEOREM 6. For $1 \leq m<\infty, E_{*}^{m}(C Y)^{+} i$ additively isomorphic to the quotient of the free strictly commutative algebra generated by the six sets

$$
\begin{gathered}
\left\{\pi Q^{s} x \mid x \in A_{r}, m \leq r-s, s \geq 0\right\}, \\
\left\{\pi \beta_{r-s} Q^{s} x \mid x \in A_{r 0}, m \leq r-s<\infty, s \geq 0\right\}, \\
\left\{\pi \mathcal{Q}^{m-r+s} Q^{s} x \mid x \in A_{r 0}, 1 \leq r-s<m, s \geq 0\right\}, \\
\left\{\pi \beta_{m} \mathcal{Q}^{m-r+s} Q^{s} x \mid x \in A_{r 0}, 1 \leq r-s<m, s \geq 0\right\}, \\
\left\{\pi Q^{t-m} R^{t-r} x \mid x \in A_{r 1}, t \geq \max (m, r+1), r<\infty\right\},
\end{gathered}
$$

and

$$
\left\{\pi \beta_{t} R^{t-r} x \mid x \in A_{r 1}, t \geq \max (m, r), r<\infty\right\}
$$

by the ideal generated by the set

$$
\left\{\left(\pi \beta_{t} R^{t-r} x\right)^{p^{t+1-m}} \mid x \in A_{r 1}, t \geq \max (m, r), r<\infty\right\} .
$$

If $p$ is odd or $m \geq 3$ the isomorphism is multiplicative. The differential in $E_{*}^{m}(C Y)^{+}$is determined by the formula

$$
\pi \beta_{m} Q^{t-m} R^{t-r} x=\left(\pi \beta_{t} R^{t-r} x\right)^{p^{t-m}}
$$

for $x \in A_{r 1}, t \geq \max (m, r), r<\infty$.

The construction of the operations is as follows. Let $M_{r}$ be the Moore spectrum $S^{-1} \cup_{p^{r}} e^{0}$ and let $K$ be the integral $K$-theory spectrum. By definition, any $x \in K_{\alpha}(X ; r)$ is represented by a stable map

$$
x: S^{\alpha} \rightarrow K \wedge \Sigma M_{r} \wedge X .
$$

Since the dual of $\Sigma M_{r}$ is $M_{r}$, such a map induces

$$
x^{\prime}: \Sigma^{\alpha} M_{r} \rightarrow K \wedge X .
$$

Applying the stable extended power functor $D_{p}$ and using the fact that $K \wedge X$ is an $H_{\infty}$ ring spectrum [3] one obtains a composite

$$
x^{\prime \prime}: D_{p} \Sigma^{\alpha} M_{r} \rightarrow D_{p}(K \wedge X) \rightarrow K \wedge X .
$$

Finally, if $e \in K_{\alpha}\left(D_{p} \Sigma^{\alpha} M_{r} ; s\right)$ for some $s$ one has the composite

$$
\Sigma^{\alpha} M_{s} \stackrel{e^{\prime}}{\rightarrow} K \wedge D_{p} \Sigma^{\alpha} M_{r} \stackrel{1 \wedge x^{\prime \prime}}{\rightarrow} K \wedge K \wedge X \stackrel{\mu \wedge 1}{\rightarrow} K \wedge X,
$$

where $\mu$ is the $K$-theory product. This composite represents an element of $K_{\alpha}(X ; s)$ depending only on $e$ and $x$. The operations $Q x, \mathcal{Q} x$ and $R x$ are obtained in this way for various choices of $e$, and the proofs of Theorems 1 , 3 , and 4 reduce in each case to the analysis of $e$. The construction has the further advantage that the proof of Theorem 5 is reduced, after some diagram chasing, to the universal case $Y=\Sigma^{\alpha} M_{r}$. Details will appear in [3]. 


\section{BIBLIOGRAPHY}

1. S. Araki and T. Kudo, Topology of $H_{n}$-spaces and $H$-squaring operations, Mem. Fac. Sci. Kyushu Univ. Ser. A 10 (1956), 85-120.

2. S. Araki and H. Toda, Multiplicative structures in $\bmod q$ cohomology theories. I, Osaka J. Math. 2 (1965), 71-115; II, Osaka J. Math. 3 (1966), 81-120.

3. R. Bruner et al, $H_{\infty}$ ring spectra, Lecture Notes in Math., Springer-Verlag, Berlin and New York (to appear).

4. F. Cohen, T. Lada and J. P. May, The homology of iterated loop spaces, Lecture Notes in Math., vol. 533, Springer-Verlag, Berlin and New York, 1976.

5. E. Dyer and R. K. Lashof, Homology of iterated loop spaces, Amer. J. Math. 84 (1962), 35-88.

6. L. Hodgkin, The $K$-theory of some well-known spaces. I. $Q S^{0}$, Topology 11 (1972), 371375.

7. _ Dyer-Lashof operations in K-theory, London Math. Soc. Lecture Notes Series, no. 11, Cambridge Univ. Press, Oxford, 1974, pp. 27-32.

8. I. Madsen, Higher torsion in SG and BSG, Math. Z. 143 (1975), 55-80.

9. J. P. May, Geometry of iterated loop spaces, Lecture Notes in Math., vol. 271, SpringerVerlag, Berlin and New York, 1972.

10. J. E. McClure and V. P. Snaith, On the K-theory of the extended power construction, Proc. Cambridge Philos. Soc. (to appear).

11. H. Miller and V. P. Snaith, On the K-theory of the Kahn-Priddy map, J. London Math. Soc. 20 (1979), 339-342.

12. — $O n K_{*}\left(Q R P^{n} ; Z_{2}\right)$, J. London Math. Soc. (to appear).

13. V. P. Snaith, Dyer-Lashof operations in K-theory, Lecture Notes in Math., vol. 496, Springer-Verlag, Berlin and New York, 1975, pp. 103-294.

Department of Mathematics, The Johns Hopkins Univeritit, Baltimore, MARYLAND 21218 